\title{
Original \\ Surgical management and prognosis of perforation secondary to typhoid fever
} Article

\author{
Sanchit Jain ${ }^{1}$, Laxmi Narayan Meena ${ }^{2}$, Prahlad Ram²
}

ABSTRACT

${ }^{I} R U H S$ College of Medical Sciences, Jaipur (Rajasthan), ${ }^{2}$ Department of General Surgery, SMS Medical College, Jaipur (Rajasthan).

Correspondence: Sanchit Jain

Email:sanchit4088@gmail.com
Introduction: Enteric perforation is a serious complication of typhoid fever and the condition has a high morbidity and mortality in many developing countries including India. No consensus exists concerning the best procedure to be performed in these cases.

Aim: The purpose of this study was to analyse our clinical experience in surgical management of enteric perforation and to determine the prognostic factors associated with morbidity and mortality.

Methods: It was a prospective study of patients who underwent surgery for typhoid intestinal perforation at SMS Medical College and Hospital, Jaipur, India, between April 2012 and October 2013. Laparotomy was performed by a midline incision. Management of perforation was based on the intraoperative findings and the procedure to be performed was decided by the operating surgeon.

Results: 88 patients were studied with a male to female ratio of 6.3:1. The mean age was 36.4 years. The peak incidence was between 21 to 30 years. All the patients presented with abdominal pain and distension. More than $90 \%$ patients showed free gas under diaphragm. $71 \%$ patients had a single perforation and $97 \%$ patients had perforations confined to ileum. Debridement with double layered closure was performed in about $58 \%$ patients, ileostomy in $25 \%$ and resection anastomosis in $18 \%$ of patients. Overall complication rate was $44.3 \%$ with surgical site infection being the most common morbidity. The mortality rate was $17.1 \%$ which was significantly affected by perforation-admission interval of more than 48 hours, number of perforations and occurrence of postoperative complications.

Conclusion: Typhoid fever leading to development of intestinal perforation continues to be a significant health problem with a high morbidity and mortality especially in rural India. The management of the disease requires an early and appropriate surgical intervention.

KEYWORDS: Enteric perforation; typhoid fever; surgical management; complications; morbidity; mortality. 


\section{Introduction}

Typhoid fever is a severe febrile illness caused primarily by the gram-negative bacillus Salmonella enteritidis serovar typhi. ${ }^{1}$ In developing countries, typhoid fever still remains a major health problem because of poor sanitary conditions. ${ }^{2}$ Among the various complications the most lethal are intestinal bleeding and ileal perforations, both arising from hyperplasia and necrosis of Peyer's patches in the terminal ileum usually occurring 2-3 weeks after the onset of the disease. ${ }^{1,3}$ Although intestinal hemorrhage is the most common complication of typhoid fever, intestinal perforation is the complication associated with highest morbidity and mortality. ${ }^{1}$ Mortality rates in cases of intestinal perforation following typhoid fever are reported to be between $5 \%$ and $62 \% .{ }^{4}$ The management of typhoid intestinal perforation poses a diagnostic and therapeutic challenge to general surgeons practicing in resource-limited countries. ${ }^{5,6}$ Surgery is considered to be the treatment of choice in these patients, who most often present late. ${ }^{7}$ However, there is no consensus regarding the best procedure to be performed in such a case. The outcome of treatment is poor in developing countries. This is due to multiple factors including delayed presentation of the disease, poor sanitation, lack of diagnostic facilities and inadequate healthcare services especially inremote areas.

The purpose of the present prospective study was to report our experience of surgical management of patients presenting with enteric perforation. This was used to evaluate the various treatment outcomes and outline prognostic factors associated with morbidity and mortality in these patients.

\section{Material and Methods}

A total of eighty-eight patients underwent surgery for enteric perforation at the Department of General Surgery, SMS Medical College and Hospital, Jaipur in the period from April, 2012 to October, 2013. This prospective study was conducted with the permission of the research and review board of the hospital.

All patients admitted to our hospital for acute pain abdomen were evaluated with detailed history of their illness (abdominal pain, abdomen distension, fever, vomiting/nausea) including the onset and duration of presenting symptoms. Significant past or personal history was also taken. After an initial general and abdominal examination (which suggested perforation peritonitis), an $\mathrm{X}$-ray chest PA view or X-ray abdomen upright view was done.The $\mathrm{x}$-ray films showed free gas under the diaphragm. In cases where a strong suspicion existed based on clinical examination but the x-ray was negative, an ultrasound of the whole abdomen was done. As soon as the diagnosis was confirmed,resuscitation was started with large volume of crystalloids (blood transfusion was done where indicated), nasogastric suction, urethral catheterization and administration of broad spectrum antibiotics, and patient was posted for surgery as soon as possible.

Peritonitis was recorded as 'general' when the whole abdomen was involved and as 'local' when limited to the lower abdomen. Patients presenting within 48 hours of onset of symptoms of peritonitis were labelled'early'while those presenting after 48 hours were called'late'. The time of typhoid intestinal perforation was subjectively determined as the time at which the patient felt an excruciating sharp pain with worsening of symptoms. Preoperative shock was defined as systolic BP less than $90 \mathrm{~mm} \mathrm{Hg}$.

Diagnosis of enteric perforation secondary to typhoid was based upon a history of fever followed by acute onset of pain in the abdomen, signs and symptoms of perforation peritonitis and a Widal test, supplemented by radiological findings of pneumoperitoneum and intraoperative findings. Intraoperative findings suggestive of perforation secondary to enteric fever include perforation on the antimesenteric border of on acutely inflamed and oedematous terminal ileum with associated peritoneal soilage. Histopathological examination of tissue biopsy from the margin of the perforation was done postoperatively.

The treatment policy in our hospital is to perform an exploratory laparotomyvia a midline incision. All dirty yellow purulent material was aspirated from peritoneal cavity and a general survey of peritoneal 
cavity was done. Based on the intraoperative findings, the various procedures performed during surgery included simple double-layered closure of perforation, resection anastomosis with or without proximal diversion stoma, loop ileostomy or resection with end ileostomy and distal mucus fistula. The decision regarding the procedure was based on the discretion of operating surgeon, number and site of perforation, condition of the bowel, degree of contamination and distance of the perforation from the ileocaecal valve. Thorough peritoneal lavage was done in every case with warm normal saline (3-5 litres).

Postoperatively patients were kept nil per oral till the time bowel sounds reappeared. Data of each patient were entered into a proforma prepared for the study. The study variables included socio-demographic data, clinical presentation, radiological findings, perforation-surgery interval, intraoperative findings (such as type of peritonitis, degree of contamination and number of perforations) and surgical procedure performed. The variables studied in the postoperative period were postoperative complications and mortality. The development of complications was noted till the time of discharge andsubsequently, infollowup every 7 days upto 3 months post - operatively.

The statistical analysis was performed using IBMSPSS software version 20. Chi-square test was used to test for the significance of association between the independent and dependent variables in the categorical data. The level of significance was considered as p-value $\leq$ 0.05 .

\section{Results}

Out of 88 patients, 76 were male (male: female ratio 6.3:1). Patients were between ages of 15 to 62 years (mean age 36.4). More than $40 \%$ were below the age of $30 \mathrm{yrs}$. All the patients acute onset of pain with abdominal distention at presentation (Table 1). 51 patients presented at admission with systolic BP below $90 \mathrm{~mm} \mathrm{Hg}$ (preoperative shock) but recovered following resuscitation with iv fluids.

Perforation-admission interval was within 48 hours (early presentation) in 17 (19.3\%) patients while remaining 71 presented after 48 hours. Generalized peritonitis was noted in 79 (89.8\%) patients while remaining 9 patients had peritonitis localized to lower abdomen.
Out of 88 patients, 82 patients $(93.2 \%)$ presented with free gas under diaphragm while remaining had a positive ultrasonography report indicated by presence of free peritoneal collection. Widal test was found to be positive in 81 patients.

Intraoperatively, a single perforation was found in 63 patients $(71.6 \%)$, two perforations in 16 patients $(18.1 \%)$ and $>2$ perforations in the remaining 9 patients (10.3\%).

Most of the perforations were confined to ileum (86 patients- 97.7\%). Three patients had perforation in jejunum while one patient also had perforation in caecum.

Debridement of margins of perforation with double layered closure was the most common procedure performed (Table 2).

A total of $39(44.3 \%)$ patients developed postoperative complications. (Table 3) Surgical site

Table 1: Symptoms at presentation

\begin{tabular}{l|l} 
Symptom & $\mathbf{N}(\%)$ \\
Abdominal pain & $88(100)$ \\
\hline Distension & $88(100)$ \\
\hline Constipation & $86(97.7)$ \\
\hline Fever & $67(76.1)$ \\
\hline Vomiting & $19(21.6)$ \\
\hline Diarrhea & $3(3.4)$
\end{tabular}

Table 2: Operative procedures performed

\begin{tabular}{l|l} 
Procedure & N (\%) \\
Debridement with double layered closure & $51(57.9)$ \\
\hline Segmental Resection Anastomosis & $16(18.2)$ \\
\hline Loop ileostomy & $13(14.8)$ \\
\hline $\begin{array}{l}\text { Resection with end ileostomy with distal } \\
\text { mucus fistula }\end{array}$ & $8(9.1)$
\end{tabular}

Table 3: Incidence of postoperative complications

\begin{tabular}{l|l} 
Complication & N(\%) \\
Surgical site infection & $26(29.5)$ \\
\hline Chest infection & $17(19.3)$ \\
\hline Intraabdominal abscess & $7(8.0)$ \\
\hline Burst abdomen & $5(5.7)$ \\
\hline Faecal fistula & $5(5.7)$ \\
\hline Stoma related complications & $4(4.5)$
\end{tabular}


infection was the most common complication. Among the patients in whom ileostomy was performed, four had complications related to stoma.

Overall mortality was 15 (17.1\%). Table 4 shows the variables which were found to be associated with prognosis.

\section{Discussion}

Intestinal perforation is the most serious complication of typhoid fever in developing countries. The incidence of the disease varies considerably in different parts of the world. ${ }^{8}$ The incidence of typhoid related intestinal perforation is an indication of endemicity of typhoid fever in any locality. ${ }^{2,9}$

Enteric perforation is more common in people of lower socio-economic status. This is probably related, poor hygiene and limited access to healthcare facilities.

In our study the number of males was much more than females with a ratio of 6.3:1 which is in concordance with most studies ${ }^{10,11}$ with the exception of Edino et al $l^{12}$ who reported a male:female ratio of $0.9: 1$. This can be explained by greater frequency of exposure due to more time spent out of home, and thus, more frequency of consumption of food that is not home-made. However some studies indicate the presence of underlying immune mechanisms and a genetic predisposition of men to typhoid fever. ${ }^{11}$

Abdominal pain with distension was the noted in all the patients. Fever was found to be less common in our patients as compared to other studies where fever was ubiquitous. ${ }^{9} 11,12$ Patients presenting after 48 hours of acute onset of severe pain (time of perforation) had a significantly higher mortality than patients who presented within two days of onset of pain. Also, preoperative shock (systolic $\mathrm{BP}<90 \mathrm{~mm} \mathrm{Hg}$ ) had a significant correlation with a higher mortality in this group.

The diagnosis of typhoid intestinal perforation was made by clinical evaluation, laboratory investigation, radiological investigations and operative findings such as perforation on antimesenteric border. ${ }^{9,11}$ First radiological investigation performed was either abdominal or chest radiograph which demonstrated free gas under the diaphragm in $93 \%$ of cases, the rate of positive x-ray finding being similar to other studies. ${ }^{10,13}$ In cases where $\mathrm{X}$-ray was inconclusive but there was a strong clinical suspicion of perforation, an abdominal ultrasonography was performed for diagnosis. For the accurate diagnosis

\section{Table 4: Variables evaluated for prediction of mortality}

\begin{tabular}{|c|c|c|c|c|}
\hline \multicolumn{2}{|l|}{ VARIABLE } & NUMBER & MORTALITY & P-VALUE \\
\hline \multicolumn{5}{|l|}{ Significant } \\
\hline \multirow{2}{*}{$\begin{array}{l}\text { Perforation-admission } \\
\text { interval }\end{array}$} & $\leq 48 \mathrm{hr}$ & 17 & 1 & \multirow{2}{*}{$<0.05$} \\
\hline & $>48 \mathrm{hr}$ & 71 & 14 & \\
\hline \multirow{2}{*}{ Preoperative SBP } & $\leq 90 \mathrm{~mm} \mathrm{Hg}$ & 51 & 13 & \multirow{2}{*}{$<0.05$} \\
\hline & $>90 \mathrm{~mm} \mathrm{Hg}$ & 37 & 2 & \\
\hline \multirow{2}{*}{ Number of perforations } & Single & 63 & 2 & \multirow{2}{*}{$<0.05$} \\
\hline & Multiple & 25 & 13 & \\
\hline \multirow{2}{*}{$\begin{array}{l}\text { Postoperative } \\
\text { complications }\end{array}$} & Present & 39 & 15 & \multirow{2}{*}{$<0.05$} \\
\hline & Absent & 49 & 0 & \\
\hline \multicolumn{5}{|l|}{ Not Significant } \\
\hline \multirow{2}{*}{ Age } & $<40 \mathrm{yr}$ & 36 & 4 & \multirow{2}{*}{$>0.05$} \\
\hline & $\geq 40 \mathrm{yr}$ & 52 & 11 & \\
\hline \multirow{2}{*}{ Sex } & Male & 76 & 12 & \multirow{2}{*}{$>0.05$} \\
\hline & Female & 12 & 3 & \\
\hline \multirow{2}{*}{ Peritonitis } & Generalized & 79 & 14 & \multirow{2}{*}{$>0.05$} \\
\hline & Localized & 9 & 1 & \\
\hline
\end{tabular}


of typhoid intestinal perforation, blood, urine and stool for culture and sensitivity are required. ${ }^{11}$ However, these tests could not be performed as the required facilities were not available. Instead Widal test was done for every patient.

Surgical intervention in typhoid perforation is considered to be the treatment of choice as it offers the best chance of patient survival. Presence of a single intestinal perforation in majority $(71.6 \%)$ of our patients is consistent with other reports. ${ }^{10,11,12}$ As seen in Table 4, patients with multiple perforations had a significantly high mortality rate compared to those with single perforations, the result being similar to other studies..$^{10,11}$

The best procedure to be performed in a case of enteric perforation is controversial. In our study the decision regarding the type of procedure was based on multiple factors as described earlier. The most commonly performed procedure was debridement with primary closure in about $58 \%$ patients. Ileostomy either loop or end was performed in 21 patients. Ileostomy is associated with multiple problems during postoperative period and also requires a second surgery for closure which may itself be associated with complications. Many previous studies also found debridement with primary closureto be the most common procedure performed. ${ }^{8,10,11,12}$ However, Sumer $e a^{4}$ reported that segmental resection followed by anastomosis was more commonly done than primary repair in their study on 22 patients. We found no correlation between surgical procedure performed and mortality rate, similar to Rahman et $a l^{2}$. This is in contrast to Shah et al ${ }^{14}$ and Athie et $a l^{15}$ who found the complications and mortality rates in resection-anastomosis patients lower than in other intervention groups. Both the authors advocated resection-anastomosis as the surgery of choice.

The most common complication in enteric perforation is surgical site infection, while the most serious is formation of a fecal fistula. ${ }^{4}$ The overall incidence of postoperative complications in our study was $44.3 \%$. This is comparable to what has been reported by others. ${ }^{410-12}$ Wound infection was the most common morbidity in postoperative period seen in 26 patients which is similar to all other studies. High rate of wound infection may be due to contamination of the laparotomy wound during the surgery. ${ }^{11}$ Five patients developed fecal fistula which closed spontaneously in the postoperative period. Four patients developed complications related to ileostomy (end/loop) which included skin excoriation with ulceration in three patients and one retraction of stoma. All these complications were managed conservatively. The development of postoperative complications had a significant correlation with mortality.

In developing countries the mortality rates range from $9-22 \%{ }^{11}$ The overall mortality rate of the present study was $17.1 \%$. Exceptionally low mortality rates of $1.5-2 \%$ have been reported from some parts of the developed world. ${ }^{16}$

We attribute the higher rate of mortality to three factors: lack of specialised care especially in rural areas, delay at the patient's end to seek treatment and failure to recognize the features of perforation peritonitis leading to late referral. On analysis of various variables associated with mortality we found perforation-admission interval of more than 48 hours, systolic BP less than $90 \mathrm{~mm} \mathrm{Hg}$ pre-operatively, multiple perforations and presence of postoperative complications being significantly associated with higher mortality.

The authors believe that a single perforation should be sutured and closed primarily either in single or double layer. In case of multiple perforations, segmental resection with anastomosis should be the preferred approach but the final decision should be based on various factors like intestinal condition, number and site of perforations, severity of peritonitis and general condition of the patient. We are of the opinion that although stoma surgery has several complications, it may be lifesaving in serious cases of typhoid perforation where the condition of the intestine as a result of typhoid disease and peritonitis precludes any kind of repair.

\section{Conclusion}

Typhoid fever leading to enteric perforation is still common in India and is associated with high morbidity and mortality. Delayed presentation, preoperative shock, multiple perforations and postoperative complications were the major predictors of mortality in this study.

Early and appropriate surgical intervention, effective preoperative and postoperative care alongwith 
use of appropriate antibiotics is thus deemed necessary for the successful management of typhoid intestinal perforation. However, the emphasis should be on strategies to prevent and reduce the incidence of enteric fever. This includes measures such as provision of safe drinking water and appropriate sewage disposal, improved personal hygiene, improving health facilities and creating health awareness among the population.

\section{References}

1. Hosoglu S, Aldemir M, Akalin S, et al. Risk factors for enteric perforation in patients with typhoid fever. Am J Epidemiol. 2004;160:46-50.

2. Rahman GA, Abubakar AM, Johnson AW, Adeniran JO.Typhoid ileal perforation in Nigerian children: an analysis of 106 operative cases. Pediatr Surg Int. 2001;17: 628-630.

3. Saxe JM, Cropsey R. Is operative management effective in treatment of perforated typhoid? Am J Surg. 2005;189:342344.

4. Sumer A, Kemik O, Dulger AC, et al. Outcome of surgical treatment of intestinal perforation in typhoid fever. World $J$ Gastroenterol. 2010;16(33):4164-4168.

5. Agbakwuru EA, Adesunkanmi AR, Fadiora SO, Olayinka OS, Aderonmu AO, Ogundoyin OO. A review of typhoid perforation in rural African hospital. West Afr J Med. 2003; 22(1):22-25.

6. Ugwu BT, Yiltok SJ, Kidmas AT, Opalawa AS. Typhoid intestinal perforation in North Central Nigeria. West Afr $J$ Med. 2005;24:1-6.

7. Talwar S, Sharma RK, Mittal DK, Prasad P. Typhoid enteric perforation. Aust NZ J Surg. 1997;67:351-353.
8. Tade AO, Olateju SO, Osinupebi OA, Salami BA. Typhoid Intestinal Perforations in a Tropical Tertiary Health Facility: A Prospective Study. East Cent Afr J Surg. 2011; 16(2):72-76.

9. Koume J, Kouadio L, Turquin HT. Typhoid ileal perforation: surgical experience of 64 cases. Acta Chir Belg. 2004;104:445-447.

10. Beniwal US, Jindal D, Sharma J, Jain S, Shyam G. Comparative study of operative procedures in typhoid perforation. Indian J Surg. 2003;65:172-177.

11. Chalya PL, Mabula JB, Koy M, Kataraihya JB, Jaka H, Mshana SE. Typhoid intestinal perforations at a University teaching hospital in Northwestern Tanzania: A surgical experience of 104 cases in a resource-limited setting. World J Emerg Surg. 2012;7:4-12.

12. Edino ST, Yakubu AA, Mohammed AZ, Abubakar IS. Prognostic Factors in Typhoid ileal Perforation: A Prospective Study of 53 Cases. JAMA. 2007;99:10431045.

13. Bansal J, Jenaw RK, Rao J, Kankaria J, Agarwal NN. Effectiveness of plain radiography in diagnosing hollow viscus perforation: study of 1,723 patients of perforation peritonitis. Emerg Radiol. 2012;19(2):115-119.

14. Shah AA, Wani KA, Wazir BS. The ideal treatment of the typhoid enteric perforation - resection anastomosis. Int Surg.1999;84:35-38.

15. Athie CG, Guizar CB, Alcantara AV, Alcaraz GH, Montalvo EJ. Twenty-five years of experience in the surgical treatment of perforation of the ileum caused by Salmonella typhi at the General Hospital of Mexico City, Mexico. Surg. 1998;123:632-636.

16. Carmeli Y, Raz R, Scharpiro JAC. Typhoid fever in Ethiopian immigrants to Israel and native - born Israelis: a comparative study. Clin Inf Dis. 1993;16:213-215. 